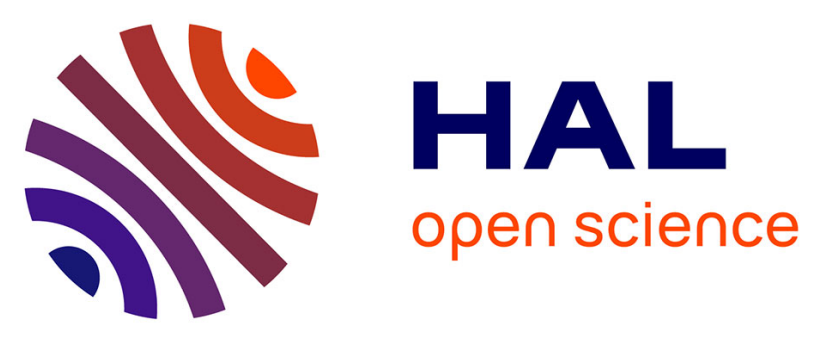

\title{
Resonant magneto-acoustic switching: influence of Rayleigh wave frequency and wavevector
}

P Kuszewski, I.S. Camara, N Biarrotte, L. Becerra, J. von Bardeleben, W. Savero Torres, A. Lemaître, C. Gourdon, J-y Duquesne, L. Thevenard

\section{To cite this version:}

P Kuszewski, I.S. Camara, N Biarrotte, L. Becerra, J. von Bardeleben, et al.. Resonant magnetoacoustic switching: influence of Rayleigh wave frequency and wavevector. Journal of Physics: Condensed Matter, 2018, 30 (24), pp.244003. 10.1088/1361-648X/aac152 . hal-01812621

\section{HAL Id: hal-01812621 \\ https://hal.sorbonne-universite.fr/hal-01812621}

Submitted on 11 Jun 2018

HAL is a multi-disciplinary open access archive for the deposit and dissemination of scientific research documents, whether they are published or not. The documents may come from teaching and research institutions in France or abroad, or from public or private research centers.
L'archive ouverte pluridisciplinaire HAL, est destinée au dépôt et à la diffusion de documents scientifiques de niveau recherche, publiés ou non, émanant des établissements d'enseignement et de recherche français ou étrangers, des laboratoires publics ou privés.

\section{(c)(1)}

Distributed under a Creative Commons Attribution| 4.0 International License 
PAPER

Related content

Resonant magneto-acoustic switching: influence of Topical Review Rayleigh wave frequency and wavevector

To cite this article: P Kuszewski et al 2018 J. Phys.: Condens. Matter 30244003

- Laser-induced magnetization dynamics and reversal in ferrimagnetic alloys Andrei Kirilyuk, Alexey V Kimel and Theo Rasing

Microwave assisted magnetic recording technologies and related physics Satoshi Okamoto, Nobuaki Kikuchi, Masaki Furuta et al.

View the article online for updates and enhancements. 


\title{
Resonant magneto-acoustic switching: influence of Rayleigh wave frequency and wavevector
}

\author{
P Kuszewski ${ }^{1}$, I S Camara ${ }^{1}$, N Biarrotte ${ }^{1}$, L Becerra ${ }^{1}$, J von Bardeleben ${ }^{1}$, \\ W Savero Torres ${ }^{2}$, A Lemaître ${ }^{3} \mathbb{D}$, C Gourdon $^{1}$, J-Y Duquesne ${ }^{1}$ \\ and $L$ Thevenard ${ }^{1}$ (D) \\ ${ }^{1}$ Sorbonne Université, CNRS, Institut des Nanosciences de Paris UMR 7588, 4 place Jussieu, 75252 \\ Paris, France \\ ${ }^{2}$ Laboratoire de Physique des Solides, CNRS, Université Paris-Sud, bât. 510, 91405 Orsay, France \\ ${ }^{3}$ Centre de Nanosciences et de Nanotechnologies, CNRS, Université Paris-Sud, Université Paris-Saclay, \\ 91460 Marcoussis, France \\ E-mail: thevenard@insp.jussieu.fr
}

Received 2 March 2018, revised 18 April 2018

Accepted for publication 30 April 2018

Published 21 May 2018

\begin{abstract}
We show on in-plane magnetized thin films that magnetization can be switched efficiently by 180 degrees using large amplitude Rayleigh waves travelling along the hard or easy magnetic axis. Large characteristic filament-like domains are formed in the latter case. Micromagnetic simulations clearly confirm that this multi-domain configuration is compatible with a resonant precessional mechanism. The reversed domains are in both geometries several hundreds of $\mu \mathrm{m}^{2}$, much larger than has been shown using spin transfer torque- or field-driven precessional switching. We show that surface acoustic waves can travel at least $1 \mathrm{~mm}$ before addressing a given area, and can interfere to create magnetic stripes that can be positioned with a submicronic precision.
\end{abstract}

Keywords: magnetoacoustics, precessional switching, surface acoustic waves, magnetic patterning, Kerr microscopy, magnetic semiconductor

S. Supplementary material for this article is available online

(Some figures may appear in colour only in the online journal)

\section{Introduction}

Resonant magnetization switching relies on triggering large angle magnetization precession by high frequency stimuli. Sub nanosecond deterministic switching was thus demonstrated using short magnetic field pulses [1-7], spin transfer torque (STT) [8-13], electric fields or ultra-short light pulses [14-16] to induce an efficient torque on the magnetization. An alternative route relies on the inverse-magnetostriction from surface acoustic waves [17-19] (SAWs). In the right conditions [20-22], they could drive resonant precessional reversal, with the noteworthy benefit that their low attenuation should enable an action remote from their generation, with wave-front shaping or stationary wave excitation to adress selectivally a given area [23]. Voltage-driven 'straintronics' also hold the promise of a lower power consumption [14, 15, 24].

In this framework, we report on the optimum conditions for acoustic magnetization reversal, and also hint to its limits. For this study, we chose to work on a dilute magnetic semiconductor, (Ga, Mn)As, in which the carrier-mediated ferromagnetic phase exhibits magnetoelasticity. Although its Curie temperature remains well below room-temperature, the tunability of its magnetic properties has proven instrumental to demonstrate key concepts in spintronics [22, 25-27].

Here we present a study of the influence of the travelling/ stationary SAW direction with respect to the magnetic easy 


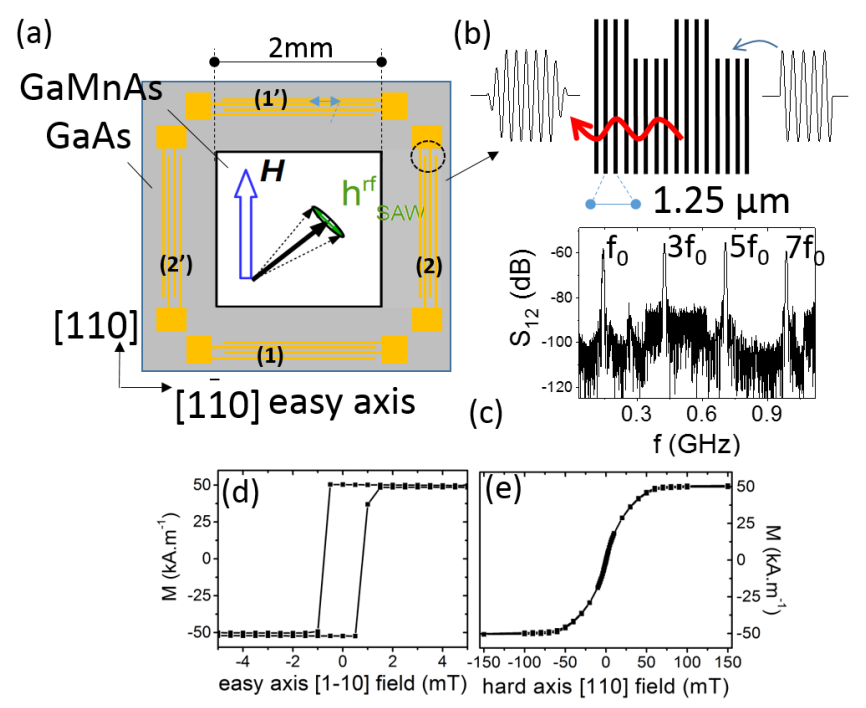

Figure 1. (a) Schematics [not to scale]: the exciting IDT 2 (resp. 1) generates a SAW travelling along [-1 10$]$ (resp. [1 10$]$ ). In the SAW-FMR regime, a small angle precession of the magnetization (black arrow) ensues, provided a hard-axis field is applied (blue arrow). (b) Schematics of the 'split 44' design of the IDT. An rf pulse excites the transducer which generates a strain pulse. (c) Gated transmission $S_{12}$ measured using a Vector Network Analyzer $\left(T=20 \mathrm{~K}, \vec{k}_{\mathrm{saw}} / /[1 \overline{1} 0]\right)$. (d)-(e) Easy and hard axis hysteresis cycles $(T=4 \mathrm{~K})$.

axis and of the SAW frequency up to about $1 \mathrm{GHz}$. We show how these govern the efficiency of the switching, but also the size and shape of the domains. In particular, homogeneous domains several hundreds of microns wide are obtained on in-plane magnetized (Ga, Mn)As, a substantial improvement over out-of-plane magnetized (Ga, Mn)(As, P) samples [22] in which only sub-micron domains were obtained. Finally, micromagnetic simulations validate the resonant switching mechanism at work, and the shape of the domains.

\section{Experimental methods}

The sample is a $h=45 \mathrm{~nm}$-thick layer of $(\mathrm{Ga}, \mathrm{Mn}) \mathrm{As}$ grown by molecular beam epitaxy on non intentionnally doped (00 01$)$ GaAs. A $16 \mathrm{~h} / 200{ }^{\circ} \mathrm{C}$ anneal results in a Curie temperature of $T_{\mathrm{C}}=120 \mathrm{~K}$ and a magnetically active Mn concentration of $x=4.9 \%$. The layer is magnetized in-plane with a uniaxial anisotropy along [ $11 \overline{1} 0]$ (see for instance the $4 \mathrm{~K}$ hard/easy axis cycles measured by superconducting quantum interference device magnetometry in figures 1(d)-(e)) . The cubic components of the anisotropy are much weaker than the uniaxial terms $[17,28]$.

The SAWs were excited electrically, using interdigitated transducers (IDTs). A $2 \times 2 \mathrm{~mm}^{2}$ (Ga, Mn)As mesa was first defined by wet etching (figure 1(a)). Four IDTs of $1 \mathrm{~mm}$ aperture were then evaporated by a $60 \mathrm{~nm}$-thick Al lift-off, using the 'split-44' transducer design [29] with 15 pairs of four equipotential digits and a nominal base periodicity of $\lambda=20 \mu \mathrm{m}$ (figure 1(b)). Relying on the natural piezoelectricity of GaAs, surface acoustic waves travelling along [110] (resp.[1 10$]$ ) were excited using IDTs 1 (resp. 2), inducing two longitudinal
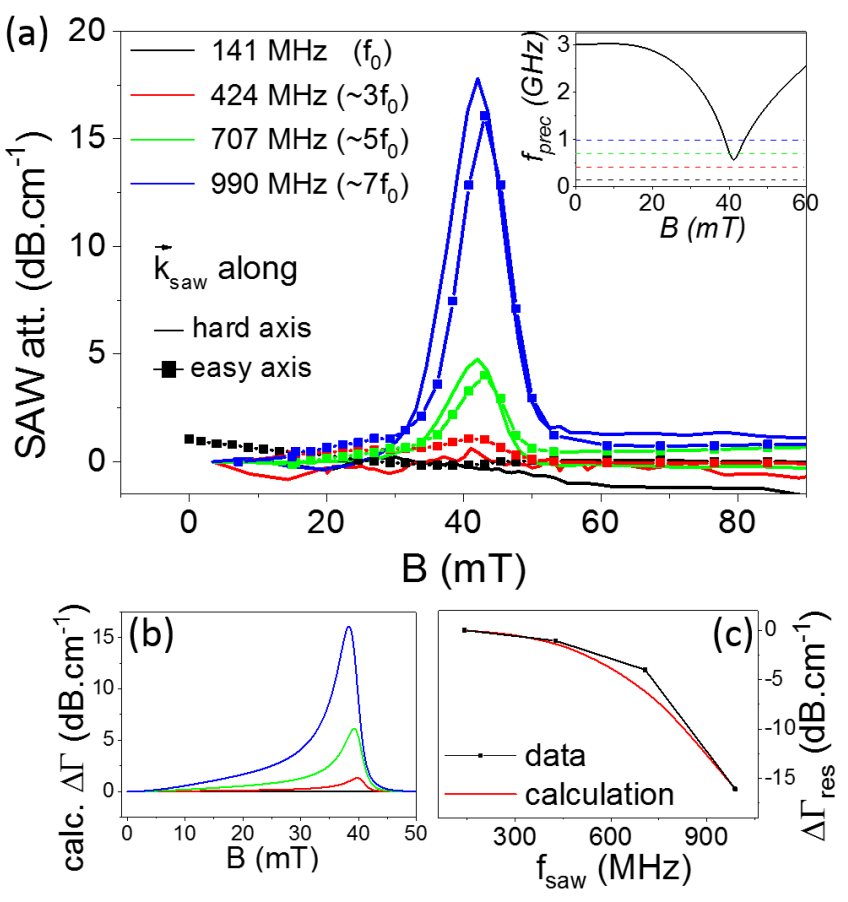

Figure 2. SAW-FMR regime: (a) Acoustic attenuation variations for a SAW propagating along the easy ([1 $\overline{1} 0])$ or hard ([1 10$])$ axis, for 4 different frequencies $(T=20 \mathrm{~K})$. The acoustic power was $16 \mathrm{~mW}$ for $5 f_{0}$ and $7 f_{0}, 50 \mathrm{~mW}$ for $3 f_{0}$ and $85 \mathrm{~mW}$ for $f_{0}$ (the different powers reflect the frequency dependency of the excitation electronic circuit). Up to these powers, corresponding to a surface strain of $\varepsilon_{x x} \approx 10^{-5}$, we observe that the attenuation and velocity variations are power independent (linear regime). The inset is a schematics of the field-dependence of the precession frequency. The resonance field lies where $f_{\text {prec }}(B)$ is closest to the first intersection with the SAW frequency (dotted lines). At the second crossing, the effective rf field generated by the SAW is almost zero [17]. (b) Acoustic attenuation variations calculated for a SAW propagating along [1 $1 \overline{1} 0]$ at $f_{0}, 3 f_{0}, 5 f_{0}$ and $7 f_{0}$ - same color coding as (a). (c) SAW attenuation variations at resonance, extracted from the data of (a) and the calculation of (b).

strain components ${ }^{4}: \varepsilon_{z z}(t)$ and $\varepsilon_{x x}(t)$, with $x / / \vec{k}_{\text {saw }}$ and $z$ normal to the sample plane. After propagating across the $(\mathrm{Ga}, \mathrm{Mn})$ As mesa, they were detected by IDTs 1' (resp. 2'). The frequency of the SAW was tuned to $n f_{0}$ with $n=1,3,5,7$ and $f_{0}=\frac{V_{r}}{\lambda} \approx 141 \mathrm{MHz}$, where $V_{r}$ is the temperature-dependent Rayleigh velocity (figure 1(c)). This design enabled us to study the influence of both the direction and the frequency of the SAW on the magnetization reversal.

Since the zero-field precession frequency of the layer-a few $\mathrm{GHz}$ as estimated from the anisotropy constants measured by cavity ferromagnetic resonance (FMR) — was systematically larger than the SAW frequencies, a field was applied along the hard axis [110] using an air-cooled CAYLAR dipole to bring the magnetization precession frequency down into resonance with the SAW (inset of figure 2(a)).

${ }^{4}$ There is also a shear $\varepsilon_{x z}(t)$ component. But it is close to zero at the surface. Within the layer, it is negligible since the layer is located just below the surface and is very thin with respect to the smallest penetration depth $\left(\approx \lambda_{7 f_{0}}=2.9 \mu \mathrm{m}\right)$. 


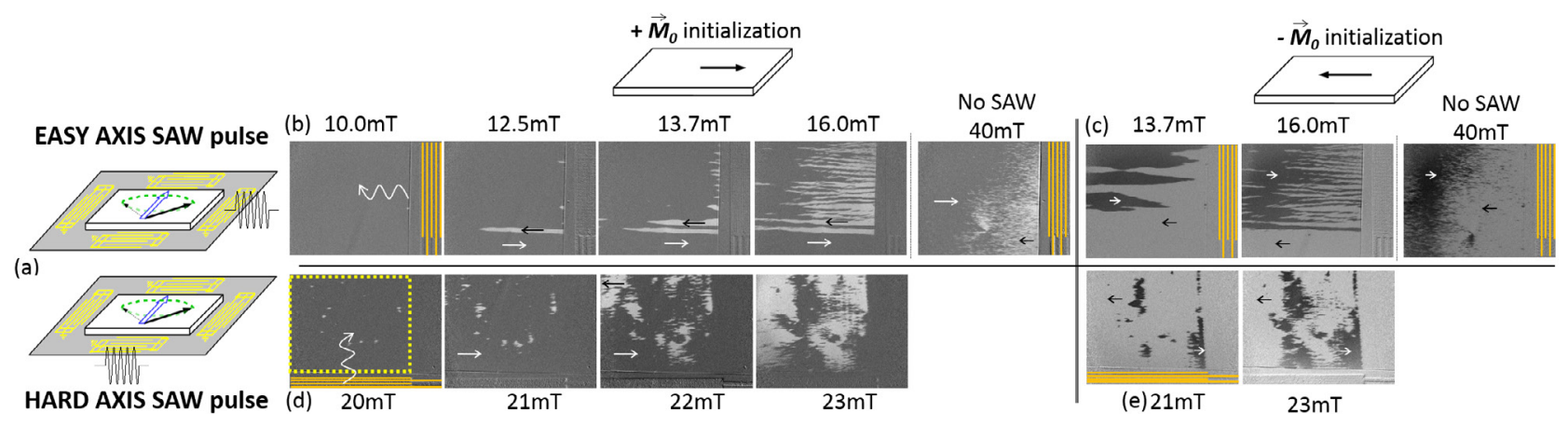

Figure 3. SAW switching regime. (a) Schematics (not to scale). At large rf power, the SAW triggers large angle precession of the magnetization (black arrow) around the applied field (blue arrow). (b)-(e) Normalized Kerr images showing the result of a single $f_{\text {saw }}=990 \mathrm{MHz} 200 \mathrm{~ns}$-long rf burst on the uniformly magnetized layer (image $306 \times 410 \mu \mathrm{m}^{2}$ ). (b), (c) $\vec{k}_{\text {saw }} / /[1 \overline{1} 0]$ (easy axis). Control images show what happens without applying any SAW pulse. (d), (e) $\vec{k}_{\text {saw }} / /[110]$ (hard axis). (b), (d) After a $+\vec{M}_{0}$ initialization, switched areas appear white. (c), (e) After a $-\vec{M}_{0}$ initialization, switched areas appear black.

\section{SAW-FMR regime}

Before using high amplitude SAWs to switch the magnetization in (Ga, Mn)As, we show that acoustic waves can excite FMR equally whether propagating along the hard or the easy magnetic axes. SAW-FMR shows up as a resonant attenuation of the wave amplitude or a resonant velocity change [17-19, 30]. To measure them, the layer is first magnetized uniformly along the easy [1 $\overline{1} 0]$ axis and 400ns-long rf bursts are sent on the exciting IDT ( $\mathbf{1}$ or $\mathbf{2}$ ) at $1 \mathrm{kHz}$ repetition frequency. The amplitude/phase of the transmitted pulse are then monitored with respect to the hard-axis field along [1 10] (see [17, 22] for a more detailed description of this time-domain SAW-FMR measurement procedure). Amplitude and phase variations were measured versus field at the four SAW frequencies for the two orthogonal travelling directions, and a large range of powers. For the sake of conciseness we will focus here on the low power $20 \mathrm{~K}$ attenuation data (figure 2(a)), sufficient to convey our message. The amplitude and velocity variations measured at other temperatures and powers were quite similar qualitatively to the data shown in $[17,22]$.

A pronounced resonance is evident in figure 2(a), as had already been seen in out-of-plane magnetized ( $\mathrm{Ga}, \mathrm{Mn}$ ) (As, P) [17] or in-plane magnetized nickel [19, 30, 31]: the SAW triggers small oscillations of the magnetization around its equilibrium position (schematics in figure 1(a)). Because the magnetic layer lies on the surface and the $\frac{h}{\lambda}$ ratio remains very small, we will consider the strain to be homogenous in the GaMnAs film, and that only the uniform precession mode can be excited. Using the cavity-FMR determined anisotropy constants, we calculate $f_{\text {prec }}(B)$ (inset of figure 2(a)) and see that the experimental resonance field is around $40 \mathrm{mT}$, for which the precession frequency stands closest to the SAW frequencies. The resonance is close to the saturation, where the $f_{\text {prec }}(B)$ curve has a steep tangent. This explains the weak variation of the experimental resonance position with $f_{\text {saw }}$. Note that a $0.2^{\circ}$ misalignement of the field with respect to the [110] axis was taken in the calculation, which will be justified in the following.

The amplitude and position of the acoustic resonance are very similar for SAWs propagating along the hard or easy axis. This can be explained by computing the effective field generated by the SAW. In the reference frame of the equilibrium magnetization [17, 19] $\mu_{0} \vec{h}_{r f}(t)=\left(0, \mp \frac{1}{2} A_{2 x y} \varepsilon_{x x}(t) \sin 2 \varphi_{0}+A_{4 \varepsilon}\left[\varepsilon_{z z}(t)-\frac{\varepsilon_{x x}(t)}{2}\right] \sin 4 \varphi_{0}\right)$, where $x / / \vec{k}_{\mathrm{saw}} / /[1 \pm 10]$. The position of the static magnetization, $\varphi_{0}$, is defined with respect to $[1 \overline{1} 0]$. Here we have introduced the uniaxial and biaxial magnetostrictive coefficients $A_{2 \varepsilon}, A_{4 \varepsilon}$ and $A_{2 x y}$ [28]: $K_{2||}=\varepsilon_{X Y, 0} A_{2 x y} M_{S}$ and $K_{2 \perp}=-M_{S}\left(A_{2 \varepsilon}-2 A_{4 \varepsilon}\right)\left(\varepsilon_{Z Z, 0}-\varepsilon_{X X, 0}\right)+\frac{\varepsilon_{X Y, 0} A_{2 x y} M_{S}}{2} . K_{2 \|}, K_{2 \perp}$ are the out-of-plane and in-plane uniaxial anisotropies, $M_{S}$ is the magnetization at saturation, and $\varepsilon_{X X, 0}, \varepsilon_{Z Z, 0}$ and $\varepsilon_{X Y, 0}$ are the static biaxial and shear strains (in the GaAs $\langle 100\rangle$ axes frame). Because $A_{2 x y} \gg A_{4 \varepsilon}$ in this material [21,28], the $A_{4 \varepsilon}$ term in $\mu_{0} \vec{h}_{r f}(t)$ is several decades times smaller than the $A_{2 x y}$ term, so the absolute value of the tickle field has a very similar field dependence (via $\varphi_{0}(B)$ ) for the two orthogonal SAW wave-vectors $\vec{k}_{\text {saw }} / /[1 \pm 10]$.

Finally, the attenuation variations at resonance increase with the SAW frequency, from barely detectable at $f_{0}$ to over $15 \mathrm{~dB} \mathrm{~cm}^{-1}$ at $7 f_{0}=990 \mathrm{MHz}$ (figures 2(a) and (c)). This is a standard SAW FMR behavior since the absorbed acoustic power varies as $P_{a b s}=-\frac{\omega_{S A W}}{2} \operatorname{Im}\left(\mathbf{h}_{\mathbf{r f}}^{*}[\chi] \mathbf{h}_{\mathbf{r f}}\right)$ where $\chi$ is the magnetic susceptilibity tensor $[19,31]$. Using the semi-infinite model developed for (Ga, Mn)(As, P) in [17], we calculate the field-dependence of the SAW attenuation variations, assuming a SAW frequency dependent filling factor $F\left(f_{\text {saw }}\right)=0.78 \frac{h}{\lambda_{\text {saw }}}$, a static shear strain $\varepsilon_{X Y, 0}=2.10^{-4}$ and a $0.2^{\circ}$ misalignment of the field with respect to the [1 10] axis (figure 2(b)). The shape and amplitude of the experimental SAW attenuation are well reproduced, as well as the frequency dependence of the attenuation at resonance (figure 2(c)). This strong dependence of the SAW attenuation with SAW frequency exists in both the linear and non-linear magnetization dynamics regimes, and will be critical for the switching efficiency.

\section{SAW-induced resonant switching}

To observe the effect of a single SAW burst on the magnetization, a longitudinal Kerr microscope, sensitive to the [1 10$]$ component of the magnetization, was used. More details on 
this set-up can be found in [32]. Normalized Kerr images are taken as follows. The sample is first initialized magnetically $+\overrightarrow{\mathbf{M}}_{0}$ or $-\overrightarrow{\mathbf{M}}_{0}$ along the easy axis and a reference image taken. Applying a constant magnetic field along [1 10 ], a single SAW burst is then emitted (figure 3(a)), chosing its duration $\tau_{\text {saw }}$ to ensure a plateau with constant strain wave amplitude (the rise/ decay time of the acoustic burst is rather long-about $85 \mathrm{~ns}$ due to the transient passage of the wave through the excitation transducer digits, see figure 1(b)). After bringing the field back to zero, a second Kerr image is taken, and divided by the reference one. The sample was reinitialized before any new SAW pulse was applied. Particular care was given to align the static bias field along the hard axis to impede domain nucleation, which could compete with the precessional reversal (hysteresis cycles are very square, with a coercive field of $2 \mathrm{mT}$ at $20 \mathrm{~K}$ at the field ramp speed used in the switching experiments). Finally, as previously discussed and quantified in references $[22,33]$ since no current is applied to the system, and thermoelasticity of GaAs is weak, no substantial temperature rise of the sample is expected from the passage of the SAW.

\section{Influence of the SAW direction on the shape of switched domains}

We first show data for a SAW burst travelling along the easy

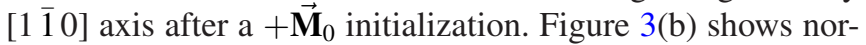
malized images taken close to the exciting IDT 2 after a single 200 ns-long burst $\left(7 f_{0}=990 \mathrm{MHz}, P_{\text {exc }}=3.5 \mathrm{~W}\right)$. This generated a surface strain component $\varepsilon_{x x}(z=0) \approx 2 \times 10^{-4}$ (see appendix A for how the SAW power and amplitude are estimated from $P_{\text {exc }}$, in the following we will drop the mention $' z=0$ '). The reversal starts at low field, $12.5 \mathrm{mT}$, and at the lower edge edge of the SAW wave-front where the displacement is higher due to the finite aperture of the transducer [34]. As the field is increased, the switching proceeds along filaments parallel to the SAW wave-vector. At $16 \mathrm{mT}$ and above, the magnetic pattern does not change anymore and is made of alternating $+\overrightarrow{\mathbf{M}}_{0}$ and $-\overrightarrow{\mathbf{M}}_{0}$ magnetized strips about 10$20 \mu \mathrm{m}$ wide, and several hundred microns long. The same experiment was then done with the SAW burst propagating along the hard magnetic axis [1 10 ] this time, parallel to the static bias field. Figure 3(d) shows images for the same SAW frequency as above, taken close to the exciting IDT 1, after a single SAW burst $\left(P_{\text {exc }}=1.5 \mathrm{~W}\right)$. The estimated SAW amplitude is a little bit smaller than above, $\varepsilon_{x x} \approx 7 \times 10^{-5}$, so that switching starts at a slightly higher field, around $20 \mathrm{mT}$. The reversed domains have a very different shape. They now form patches or columns parallel to the wave-vector, with jagged edges. At $23 \mathrm{mT}$, domains several hundreds of $\mu \mathrm{m}^{2}$ have reversed on the SAW path.

In both configurations, the switching pattern does not depend on the SAW pulse duration, as long as it is longer than the transient regime. For shorter pulses, reversal persists in the (magnetically) weaker parts of the sample. It is observed at all temperatures up to $T_{\mathrm{C}}$ at decreasing fields, as expected from the temperature dependence of the resonance [17]. No reversal was observed out of the SAW path, or when the IDT was excited out of its resonance. Monitoring the SAW amplitude

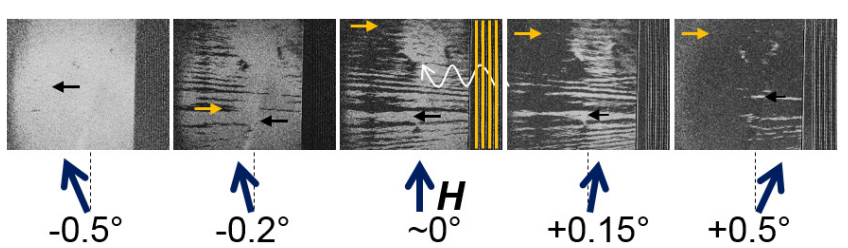

Figure 4. Final magnetization position after a $400 \mathrm{~ns}$ long pulse, depending on the misalignment of the magnetic coil with the [1 10$]$ direction, taken as the $0^{\circ}$ position $(31 \mathrm{mT}$ bias field, $T=20 \mathrm{~K}$, $\vec{k}_{\text {saw }} / /[1 \overline{1} 0]$, images $\left.306 \times 410 \mu \mathrm{m}^{2}\right)$.

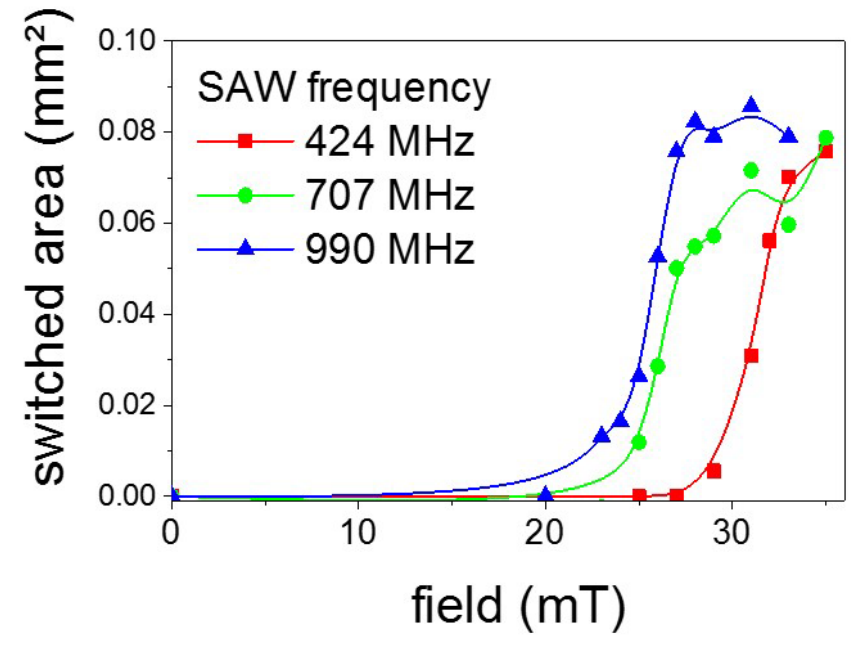

Figure 5. Switched magnetization area in front of IDT $\mathbf{1}$ after a 200 ns SAW burst $\left(T=20 \mathrm{~K}, \vec{k}_{\text {saw }} / /[110]\right.$, averaging area is the dotted rectangle in figure 3(d)). The rf excitation power is adjusted for a constant strain $\varepsilon_{x x}(z=0) \approx 7 \times 10^{-5}$. Switching at $f_{0}=141 \mathrm{MHz}$ is too inefficient at this SAW amplitude to appear on this graph. The field was misaligned by a fraction of a degree, so that at high field, almost $100 \%$ of the area has switched.

versus field in both configurations shows that it now resonates lower than the $40 \mathrm{mT}$ observed in the linear regime (figure 2(a)): $25 \mathrm{mT}$ for $P_{\text {exc }}=3.5 \mathrm{~W}$ when $\vec{k}_{\text {saw }} / /[1 \overline{1} 0]$ and $30 \mathrm{mT}$ for $P_{\text {exc }}=1.5 \mathrm{~W}$ when $\vec{k}_{\text {saw }} / /\left[\begin{array}{lll}1 & 1 & 0\end{array}\right]$. This power-dependent downshift of the resonance had already been observed in out-of-plane magnetized $(\mathrm{Ga}, \mathrm{Mn})(\mathrm{As}, \mathrm{P})$ [22] and is expected in the non-linear regime of magnetization precession, regardless of what drives it, be it an rf field [35] or spin transfer torque [36].

SAW switching also works after a $-\overrightarrow{\mathbf{M}}_{0}$ initialization (figures 3(c) and (e)). At low field, although the switched domains have the same overall shape, they are not located in the same region of the sample nor cover the same area as when starting from $+\overrightarrow{\mathbf{M}}_{0}$ (see the images at $13.7 \mathrm{mT}$ for $\vec{k}_{\text {saw }} / /[1 \overline{1} 0]$ and 21 $\mathrm{mT}$ for $\vec{k}_{\mathrm{saw}} / /[110]$ in figures 3(b) and (d)). Likewise, only about half the observed zone has switched on average at high field for $\vec{k}_{\text {saw }} / /[1 \pm 10]$. The final switching pattern starting from $+\overrightarrow{\mathbf{M}}_{0}$ is complementary to the one starting from $-\overrightarrow{\mathbf{M}}_{0}$ : only the areas unswitched starting from $+\overrightarrow{\mathbf{M}}_{0}$ have switched. The unfortunate consequence of this is that magnetization cannot be toggled from $\pm \overrightarrow{\mathbf{M}}_{0}$ to $\mp \overrightarrow{\mathbf{M}}_{0}$ by long SAW pulses. A possible explanation for this is a weak variation of the precise alignment of the field along [1 110$]$ across the image.

To test the latter, we first do a control experiment where the magnetization is initialized $+\overrightarrow{\mathbf{M}}_{0}$ or $-\overrightarrow{\mathbf{M}}_{0}$, the field 
(a) Stationary SAWs along [110]

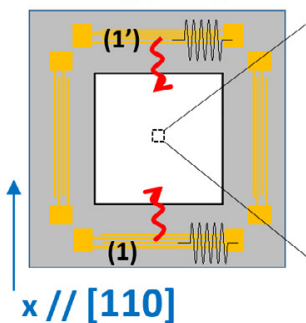

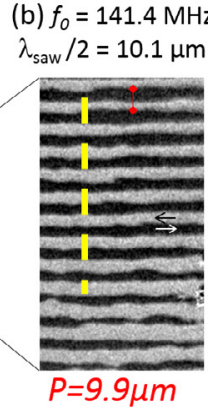

(c) $3 f_{0}=424 \mathrm{MHz}$

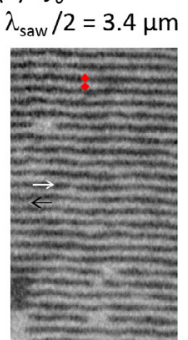

$P=3.5 \mu \mathrm{m}$ (d) $5 f_{0}=707 \mathrm{MHz}$

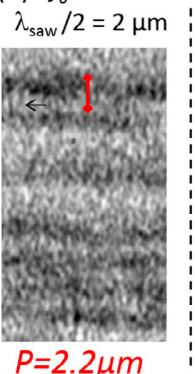

(e) Stationary SAWs along [010]

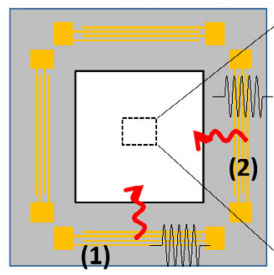

(f) $f_{0}=141.4 \mathrm{MHz}$ $\lambda_{\text {saw }} / \sqrt{ } 2=14.1 \mu \mathrm{m}$

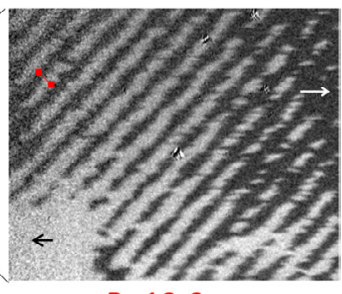

$P=13.9 \mu \mathrm{m}$

Figure 6. (a) Excitation of stationary SAWs along [1 10$](T=40 \mathrm{~K})$. Close-up of the switched magnetization pattern between IDTs 1 and 1' after a $400 \mathrm{~ns}$-long SAW burst: (b) $f_{0}=141 \mathrm{MHz}\left(B=20 \mathrm{mT}, P_{\text {saw }}=13 \mathrm{~mW}\right)$, (c) $3 f_{0}=424 \mathrm{MHz}\left(B=20 \mathrm{mT}\right.$ and $\left.P_{\text {saw }}=13 \mathrm{~mW}\right)$, and (d) $5 f_{0}=707 \mathrm{MHz}\left(B=18 \mathrm{mT}, P_{\text {saw }}=1.2 \mathrm{~mW}\right)$. The expected $\lambda_{\text {saw }} / 2$ periodicity (black font) is calculated using $\lambda_{\text {saw }}=\frac{V_{r}}{f}$ and $V_{r}=2852 \mathrm{~m} \mathrm{~s}^{-1}$, the experimental one appears in red font and lines. The dashed yellow line is a typical line scan used to make the color maps of figure 7. (e) Excitation of stationary SAWs along [0 10]. (f) Switched magnetization pattern between IDTs 1 and 2 after a $300 \mathrm{~ns}$ long SAW burst at $f_{0}=141 \mathrm{MHz}\left(B=24 \mathrm{mT}\right.$, image $\left.158 \times 205 \mu \mathrm{m}^{2}\right)$. For $f_{0}$ and $3 f_{0}$ excitation frequencies, the magnification of the microscopy set-up was $\approx 20(1$ pixel $=0.295 \mu \mathrm{m})$, for $5 f_{0}$ it was $43(1$ pixel $=0.15 \mu \mathrm{m})$.

ramped up to $40 \mathrm{mT}$, and then brought back down to zero, without having applied any SAW pulse (figures 3(b) and (c), 'NO SAW' images). If the magnetization is brought fully along [1 10 ] under this field, we expect a collection of equiprobable $+\overrightarrow{\mathbf{M}}_{0}$ and $-\overrightarrow{\mathbf{M}}_{0}$ domains at remanence. The normalized images clearly show that this only occurs on one part of the image or another, depending on the initial magnetization, and that these regions correspond precisely to those switching under SAW. This could be well be explained by the existence of slightly curved field lines: if the sample is not exactly half-way between the coil poles, the field could be slightly offset to the right of [110] on the left part of the image, and to the left of [110] on the right part. Secondly, the angle of the coil was varied in a controlled fashion. The resulting percentage of averaged switched magnetization goes from $50 \%$ to $100 \%$ with a disalignment of a fraction of a degree towards the $+\overrightarrow{\mathbf{M}}_{0}$ or $-\overrightarrow{\mathbf{M}}_{0}$ directions (figure 4). Hence SAW-driven resonant switching is extremely sensitive to the precise alignment of the magnetic coil, as already shown experimentally and numerically on out-of-plane $(\mathrm{Ga}, \mathrm{Mn})(\mathrm{As}, \mathrm{P})$ [22]

\section{Influence of the SAW frequency on the switching efficiency}

We now show how the efficiency of the switching varies with SAW frequency. This was quantified by averaging the switched area in front of the exciting transducer (dotted rectangle in figure $3(\mathrm{~d})-20 \mathrm{mT}$ ). Great care was taken to work at constant SAW amplitude, and constant magnetic field angle. We show for instance the resulting efficiency plot (figure 5) for $\vec{k}_{\text {saw }} / /\left[\begin{array}{ll}1 & 10\end{array}\right]$, and a constant surface strain of $\varepsilon_{x x} \approx 7 \times 10^{-5}$. Reversal clearly occurs earlier in field with increasing SAW frequency. This is consistent with the increase of the SAW attenuation with $f_{\text {saw }}$ (shown in the linear regime in figure 2(a), and similar behavior at high acoustic powers): the power is lost by the SAW to the magnetization, which fuels an efficient reversal. An identical behavior was

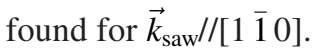

\section{Stationary SAWs: influence of $f_{\text {saw }}$ on magnetic patterning period}

The data presented up to now has relied solely on propagating acoustic waves. Yet the use of stationary waves can be relevant for magnetic patterning, as was shown on FeGa by $\mathrm{Li}$ et al [23] ( $\left.f_{\text {saw }}=158 \mathrm{MHz}\right)$. The mechanism at work was not detailed in this paper, but probably implied the reduction of domain nucleation/propagation barriers [33] rather than precessional switching. Using the different available SAW frequencies of our device, we study the effect of $f_{\text {saw }}$ in resonant switching induced by stationary SAWs along [1 10$]$. For this, IDTs 1 and 1' are excited simultaneously and the magnetization is observed half-way between them (figure 6(a)). The applied field was chosen high enough to have larges patches of homogeneously reversed magnetization.

Figures 6(b)-(d) show a close-up of the center of the sample after a single 400ns-long burst on each transducer at $f_{0}, 3 f_{0}$ and $5 f_{0}$. For each of those frequencies, the stationary SAW clearly imprinted a $\lambda_{\text {saw }} / 2$-periodic pattern to the switched magnetization. This can be explained as follows: for two counter-propagating SAWs of amplitude $A$ and relative phase $\phi$, the stationary wave has an amplitude of the form $A_{110}=2 A \cos \left(\omega_{\text {saw }} t+\phi\right) \cos \left(\frac{2 \pi}{\lambda_{\text {saw }}} x+\frac{\phi}{2}\right)$. Because the SAW burst is much longer than a single period, switching occurs when the SAW amplitude reaches a particular threshold in absolute value [22], yielding the $\lambda_{\text {saw }} / 2$ periodicity of $\left|A_{110}\right|$ in the magnetic pattern (figures 6(b)-(d)). Note that the micron-resolution of our microscope prevented us from seeing the stationary pattern at $7 f_{0} \approx 990 \mathrm{MHz}$ (fringe width $\left.\lambda_{\text {saw }} / 4=0.7 \mu \mathrm{m}\right)$.

Likewise, magnetic fringes along [100] can be patterned by exciting simultaneously two perpendicular SAWs (IDTs 1 and 2, figure 6(e)). This time the amplitude of the stationary wave reads $A_{100}=2 A \cos \left[\frac{\pi}{\lambda_{\text {saw }}}(x+y)-\omega_{\text {saw }} t+\phi\right]$ $\cos \left[\frac{\pi}{\lambda_{\text {saw }}}(x-y)+\phi\right]$. The $\frac{\lambda_{\text {saw }}}{\sqrt{2}}$-periodicity along [1 00$]$ expected from $\mid A_{100} l$ is evident in figure 6(f). A SAW wave-

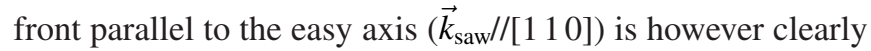


the more favorable configuration since the resulting magnetic pattern presents a weak magnetostatic cost, the charges being comfortably sent to the extremities of the fringes. This is less true when at least one of the travelling SAW wavefront is perpendicular to the easy axis as in figure 6(f), where a slight horizontal smudging of the fringes can be seen along [1-10].

Finally, changing the relative phase of the exciting bursts, one can also impose the position of the magnetic pattern. This was done for $f_{0}$ and $3 f_{0}$ (fringes parallel to $[1-10]$ ). The two bursts arriving on IDTs $\mathbf{1} / \mathbf{1}^{\prime}$ have been delayed in steps of $\delta \phi=11.5^{\circ}$ using a mechanical phase-shifter. Before each of those steps, the sample was reinitialized $+\overrightarrow{\mathbf{M}}_{0}$ and a field applied along the hard axis. After switching for a given $\phi$ value, the intensity profile of the fringes along [1 10$]$ was plotted, and the low-frequency background removed. A $\delta \phi$-interpolated color map was then created with this data (figure 7). The $\lambda_{\text {saw }} / 4$ width of a single fringe clearly appears in this graph, as well as the $\lambda_{\text {saw }} / 4$ shift (half-period of the pattern) for a $180^{\circ}$ dephasing. An important consequence ensues from this experimental result: while resonant switching using stationary SAWs is probably not the most adapted to imprint very small magnetic patterns, the wave-like nature of the switching implies that a very precise, nanometric positionning of these patterns could easily be implemented, an appealing proprety for reconfigurable magnetic memories.

\section{Compatibility of multi-domain formation with precessionnal switching mechanism}

Now that a clear experimental demonstration of SAW-driven switching of in-plane magnetization has been presented, we focus on the final multi-domain configuration that was obtained (figure 3), and whether it is compatible or not with a resonant switching mechanism.

In the simplified macrospin theory of precessional switching, when starting from a given initial magnetization $\pm \overrightarrow{\mathbf{M}}_{0}$, switching to $\mp \overrightarrow{\mathbf{M}}_{0}$ should only be possible if the duration of the effective pulse field is an odd-multiple of half the precession frequency under the applied bias field [21]. This feature of precessional switching was beautifully demonstrated experimentally on small metallic devices (typically $100 \mathrm{~nm}-10 \mu \mathrm{m}$-wide ellipses) for very short excitation pulses (electric [14] or magnetic fields [9], or current [11]). Beyond a few nanoseconds duration and for larger samples however, coherence was destroyed. Spatial and temporal dephasing of the precession was due to thermal effects (via the stochastic nature of the begining of the precession), or domain wall creation [4, 6, 11, 37] (for bigger structures), rather than chaotic magnetization trajectories [10]. This lead to the switched/ unswitched probability rapidly converging towards $50 \%$ with excitation pulse duration. We expect similar phenomena to occur when driving precessional switching acoustically, with the dephasing caused both by the spread in anisotropy constants in the magnetic layer and the relatively long duration of the excitation pulses.

Elegant analytical solutions have been derived for field and STT-driven precessional reversal of spin valves or

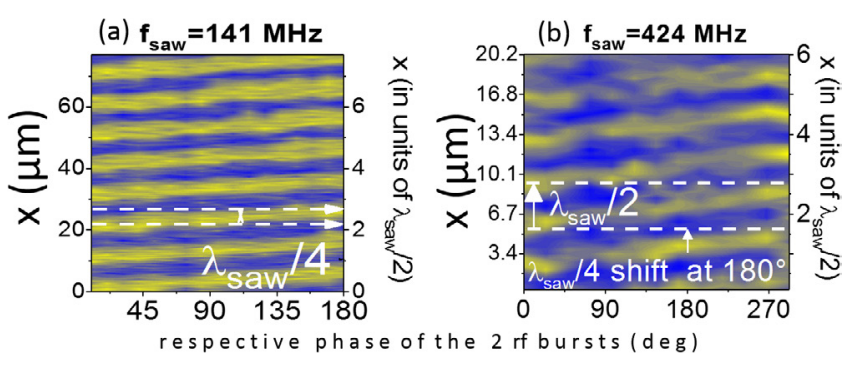

Figure 7. Maps of magnetic contrast along [1 10] versus relative phase of the $400 \mathrm{~ns}$ rf bursts on IDTs $\mathbf{1 / 1}$ ' $(T=40 \mathrm{~K})$. The left axis is the $x$-coordinate along [1 10 ] in microns. The right axis is its normalization by $\lambda_{\text {saw }} / 2$ indicated in figure $6 . f_{0}=141 \mathrm{MHz}$ ( $\left.B=20 \mathrm{mT}, P_{\text {saw }}=6.3 \mathrm{~mW}\right)$. (b) $3 f_{0}=424 \mathrm{MHz}(B=26 \mathrm{mT}$, $\left.P_{\text {saw }}=4 \mathrm{~mW}\right)$.

magnetic tunnel junctions [38-41]. In order to keep calculations tractable, they are however often limited to idealized ellipsoid geometries. Being moreover macrospin calculations, they do not take into account exchange interaction and dynamic demagnetizing effects, which are expected to have a central role in the final switched domain configuration of our system. We therefore implement instead micromagnetic $\mathrm{MuMax}^{3}$ simulations [42]. We first assumed no dispersion of the native uniaxial anisotropy constant $K_{2 \|}$. The effect of the propagating SAW on the system is modelled as: $K_{2 \| \mid}(x, t)=K_{2 \|}+\Delta K_{2 \| \mid} \cos \left(k_{\text {saw }} x-\omega t\right.$ ) (see appendix $\mathrm{B}$ for technical details on the simulations, and figure 8(a)top for snapshots of the anisotropy). $\Delta K_{2||}$ is proportional to the SAW amplitude and to the magneto-elastic coefficient $A_{2 x y}$. Starting from a uniform $+\overrightarrow{\mathbf{M}}_{0}$ configuration, we apply a $20 \mathrm{mT}$ magnetic field along the hard axis. Figure 8(a)-bottom shows selected snapshots of the time evolution of the magnetization (see the full 'M(t) no dispersion.avi' movie in the supplementary information (stacks.iop.org/JPhysCM/30/244003/ mmedia)). As expected from macrospin calculations [21], the magnetization follows a wide angle precession around the vertical (hard axis) direction. Once the SAW emission is stopped $(t=12.12 \mathrm{~ns})$, the acoustic pulse finishes propagating across the simulation window, and the magnetization returns to a small damped precession around its final equilibrium position. For $\tau_{\text {saw }}=12 T_{\text {saw }}$, the final state after removal of the field is a fully switched uniform magnetization $-\overrightarrow{\mathbf{M}}_{0}$, even though exchange and demagnetization energy costs have been taken into account. Moreover, for this magnetic field/SAW amplitude combination guarantying large amplitude oscillations of $\overrightarrow{\mathbf{M}}(\vec{r}, t)$, the final state can be chosen by how many multiples of $T_{\text {saw }}$ have elapsed during $\tau_{\text {saw }}$ (figure 9(a)). The remanent state depends quite subtly on the detuning between the SAW frequency and the precession frequency [22].

When $1 \%$ fluctuations are included in the magneto-elastic constants, a very different behavior is observed (figures 8(b) and (c) and full ' $\mathrm{M}(\mathrm{t})$ dispersion-SAW along easy/hard axis. avi' movies in the supplementary information). After a few SAW periods, the precession amplitude starts to vary across the SAW wavefront, introducing a progressive dephasing that is amplified as the SAW progresses. The spatial variations of the magneto-elastic constants reflect on the local efficiency of the SAW rf field. This triggers the nucleation of pockets 

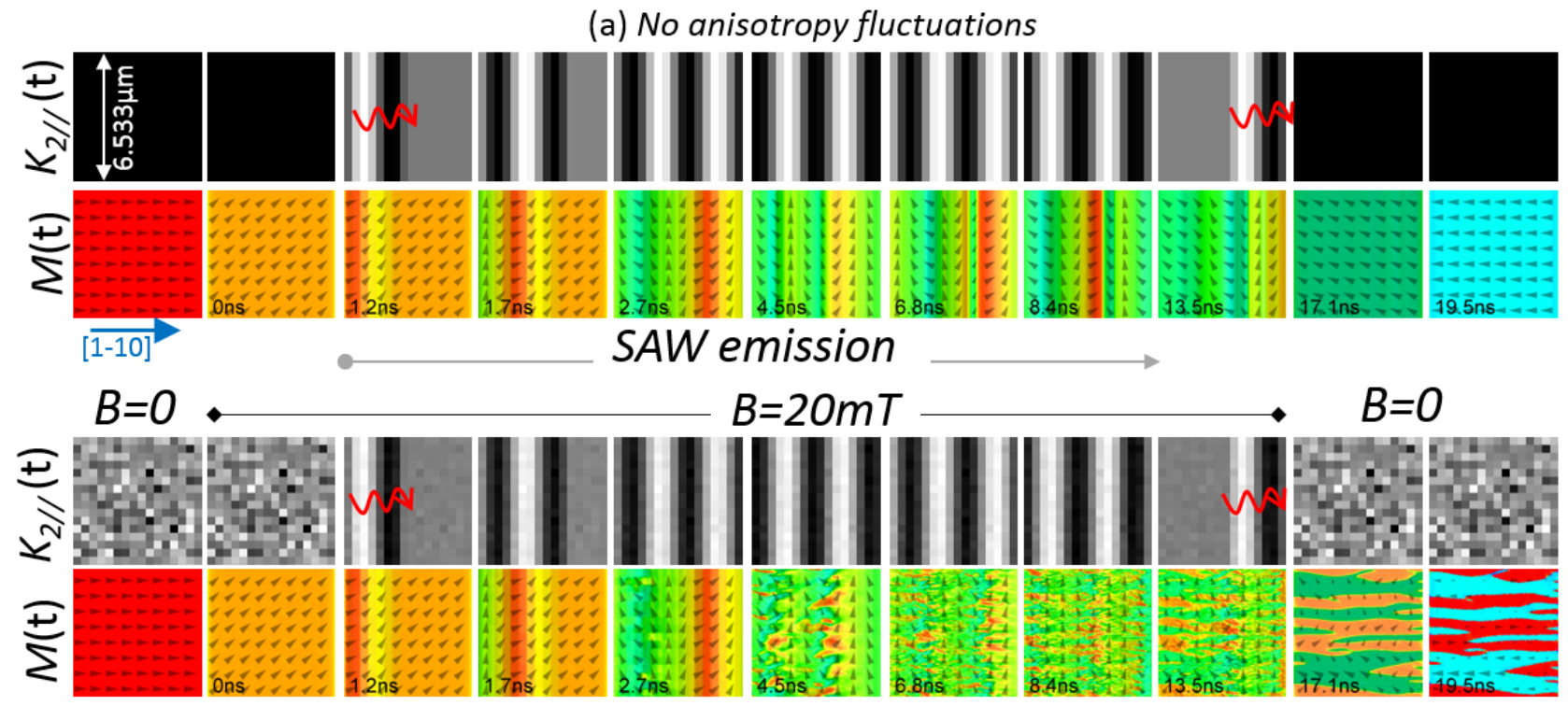

(b) $1 \%$ anisotropy fluctuations, $\boldsymbol{k}_{\text {saw }} / /$ easy axis

(c) $1 \%$ anisotropy fluctuations, $\boldsymbol{k}_{\text {saw }} \perp$ easy axis

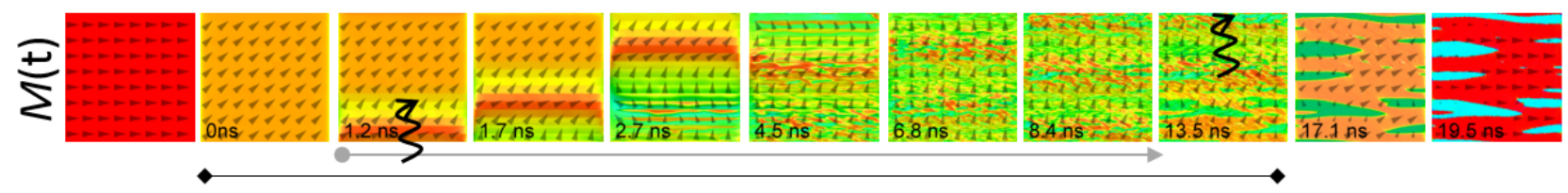

Figure 8. Micromagnetic simulations of SAW-driven resonant magnetization reversal $(B=20 \mathrm{mT}$, other parameters indicated in appendix $\mathrm{B}$, magnetization color wheel in the bottom right-hand side). The simulation window is $2.3 \lambda_{\text {saw }}$ wide. The SAW is emitted from the left between $t=0.1$ and $12.12 \mathrm{~ns}\left(12 T_{\text {saw }}\right)$. The field is turned on between $t=0$ and $17 \mathrm{~ns}$ (see full movies in the supplementary information).

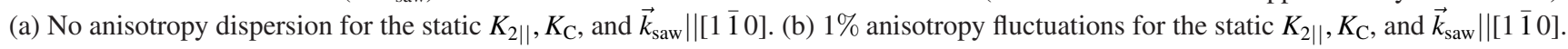
The top panel is a normalized map of the time- and space- dependent uniaxial anisotropy modulated by the SAW. The bottom panel is the state of the magnetization at the corresponding time. (c) Same as (b), but with $\vec{k}_{\text {saw }}||\left[\begin{array}{ll}1 & 1\end{array}\right]$.
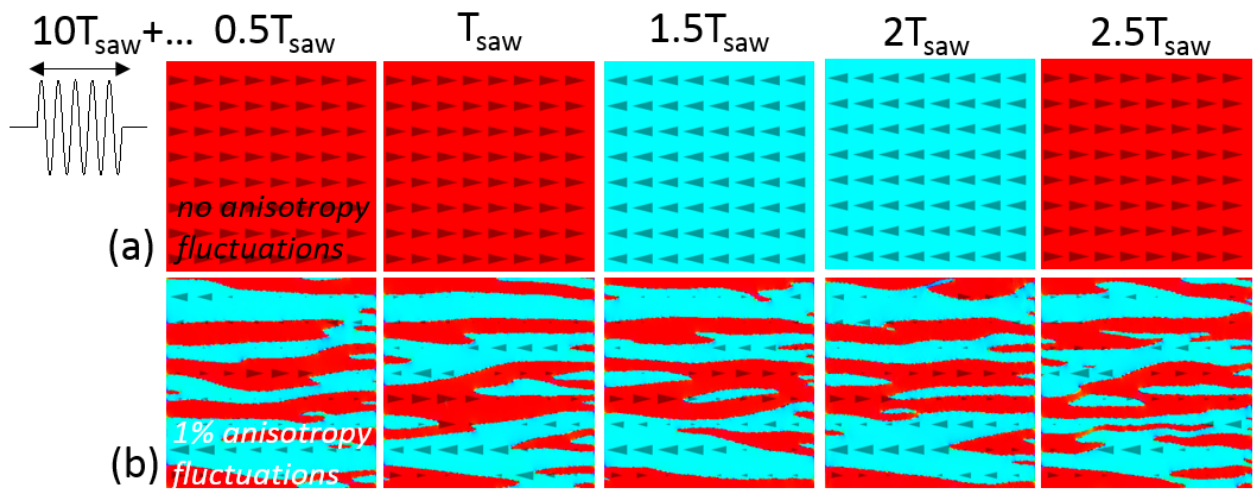

Figure 9. Effect of the SAW pulse duration $\tau_{\text {saw }}=10-12.5 T_{\text {saw }}$ on the final remanent state, starting from $+\overrightarrow{\mathbf{M}}_{0}\left(\vec{k}_{\text {saw }} \|[1-10], B=20 \mathrm{mT}\right.$, other parameters indicated in appendix B). Red (resp. blue) areas have not switched (resp. switched) to $-\overrightarrow{\mathbf{M}}_{0}$ : (a) No anisotropy dispersion for $K_{2 \|}, K_{\mathrm{C}}$ : the switching is $2 T_{\text {saw }}$-periodic as expected from the macrospin approach. (b) $1 \%$ anisotropy fluctuations for $K_{2 \|}, K_{\mathrm{C}}$. A minor dispersion in the anisotropy creates a multi-domain structure that weakly depends on the SAW pulse duration.

of anomalies, which the SAW propagation then streches out into characteristic filaments. When the field is switched off $(t=17 \mathrm{~ns})$, exchange and magnetostatic energies govern the formation of clear micron-sized fully switched (blue) or unswitched (red) domains. The system has limited the formation of charged transverse domain walls along [1 10 , preferring [43] the less costly Néel walls along [1 $1 \overline{1} 0]$. This results in filamentation along the the easy axis. Varying the SAW pulse duration changes only weakly the final state (figure 9(b)), contrary to the case when no anisotropy fluctuations are included. The exact same simulation can be run

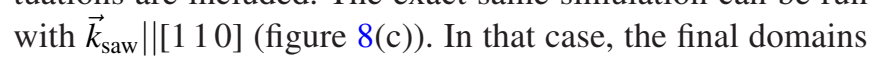


are larger and less filamented than for the $\vec{k}_{\text {saw }} \|[1 \overline{1} 0]$ case, since the SAW wave-front evolves parallel to the magnetic easy axis, a much more confortable situation from the point of view of magneto-statics.

We need to underline that the code implemented for the MuMax simulations imposes an identical spatial grid (here with a super-cell of $408 \mathrm{~nm}$ ) to describe the SAW propagation and the anisotropy fluctuations, so that large scale anisotropy fluctuations (e.g. region size of a few microns) cannot be combined with a fine description of the SAW (e.g. region size below $\lambda_{\text {saw }} / 20$ ). This is possibly why the characteristic micron-width of the filaments found in the simulation is smaller than the one observed experimentally (around 10-20 $\mu \mathrm{m})$. A full study of the critical dispersion scale to SAW wavelength ratio leading to filamentation is therefore out of the scope of the present paper. Yet, the simulations reproduce qualitatively very well three experimental features (figure 3): (i) the final state is not uniformly magnetized, but a collection of $+\overrightarrow{\mathbf{M}}_{0}$ and $-\overrightarrow{\mathbf{M}}_{0}$ domains. For $\vec{k}_{\text {saw }} \|[1 \overline{1} 0]$, these take the form of filaments running roughly parallel to $\vec{k}_{\text {saw }}$, (ii) the final state is very weakly dependent on the SAW pulse duration, (iii) the switched domains are broader and less filamented when the SAW travels along the hard axis rather than along the easy axis.

\section{Conclusion}

We have presented a comprehensive investigation of resonant magnetic switching by a surface acoustic wave with a special emphasis on the role of the SAW frequency and the interplay between the SAW wavevector direction and the easy magnetic axis. The main results are the demonstration that: (i) resonant reversal of in-plane magnetization is possible using large amplitude Rayleigh waves travelling along the easy or hard magnetic axis. The main difference between the two geometries lies in the aspect ratio of the reversed domains, with the magnetization clearly trying to reduce its magnetostatic energy by forming jagged or pointy domains along the easy axis. (ii) The reversed domains are several hundreds of $\mu \mathrm{m}^{2}$, much larger than previous demonstrations of STT- or fielddriven precessional switching. This is also in stark contrast with SAW-induced switching in out-of-plane magnetized $(\mathrm{Ga}, \mathrm{Mn})(\mathrm{As}, \mathrm{P})$ on which both the large spread of magnetic anisotropy and the natural tendency to self-organization into up/down domains meant that the switching occurred over small, sub-micron areas [22]. (iii) When the SAW wavefront lies parallel to the easy axis, the switched domains are large enough for periodic magnetic patterns to be carved out in stationary geometry, with a sub-micron positionning precision. There is no restriction to the minimum magnetic period that can be reached, save that of being able to excite high frequency SAWs (and to be able to observe sub-micron features). (iv) Micromagnetic simulations clearly confirm that the formation of multi-domains and the lack of pulse-length dependence of the final switched state are both compatible with a resonant precessional mechanism.
The limitations of this switching scheme have also been evidenced. The final pattern appeared very sensitive to field alignment and spatial variations of the anisotropy. This has also been one of the bottlenecks of precessional switching of magnetic tunnel junction or spin-valves, for which the asymmetry in the switching probabilites between parallel and anti-parallel configurations was due to the current-generated Oersted field or the stray field from the polarizing/analyzing layers [6, 4446]. Several strategies were implemented in these systems to bypass this issue, such as the use of a synthetic antiferromagnet perpendicular polarizer to limit spurious stray fields. In the SAW switching scheme, a possible route would be to engineer the magnetic anisotropy to have the zero-field precession frequency close to $f_{\text {saw }}$.

The long rise time of the SAW pulse-imposed by the large number of teeth required to excite efficiently the chosen frequency - is also likely detrimental to the determinism of the switching. The optimal design for SAW switching would therefore include a single digit-pair transducer (very short transient regime), with a SAW emission centered around a high frequency (high efficiency), and a uniaxial layer structured into submicron devices. This should allow to chose the final switched state whilst maintaining the advantages of propagating/stationary wave to adress selectivally a structure spatially.

\section{Acknowledgments}

This work has been supported by the French Agence Nationale de la Recherche (ANR13-JS04-0001-01). The authors acknowledge S Majrab (INSP) for technical assistance and $\mathrm{H}$ Krenner (Augsburg University) for fruitful discussions.

\section{Appendix A. Working at constant SAW amplitude}

We have recently developed two precise techniques to estimate the SAW amplitude, using x-ray diffraction [47] or a vector network analyser [48]. The former is difficult to implement at cryogenic temperatures. The latter relies on measurements of the $S$ parameters and modelling using the coupling-of-modes theory. It could however not be implemented here: (i) no 'dip' was observed in the $\left|S_{i i}\right|^{2}$ reflection coefficients, and (ii) working at cryogenic temperatures made it quite complicated to evaluate precisely the contribution of the fixtures, an indispensable step in this approach. For this reason, we proceeded to a gross estimate of the acoustic power as follows. Exciting IDT $\mathbf{i}$ with an rf voltage of amplitude $U_{\text {exc }}$, we detect an echo of amplitude $U_{\text {det }}$ on the opposite transducer i'. The incident electrical power $P_{\text {exc }}$ is first measured on a matched load $(50 \Omega)$. The echo amplitude is then measured on a $50 \Omega$ load and converted to an electrical power $P_{\text {det }}$. Assuming an equal transduction coefficient $\beta$ for both IDTs, we get $P_{\text {det }}=\beta^{2} P_{\text {exc }}$, from which $\beta$ is computed. Finally, the acoustic power is estimated as $P_{\text {saw }}=\beta P_{\text {exc }}$. The expression of the Poynting vector then yields the numerical value of the strain [34]. Note that this was performed at low temperature and in zero field (i.e. far from any magnetic resonance capable of damping the SAW 
amplitude, see figure 2(a)). For the device presented here, the transduction insertion loss amounted to $-25 \mathrm{~dB}$ to $-30 \mathrm{~dB}$, depending on the frequency, the IDT and the temperature.

\section{Appendix B. Micromagnetic simulations parameters}

The size of the simulation is $6533 \times 6533 \times 50 \mathrm{~nm}$ $\left(L_{x} \times L_{y} \times L_{z}\right)$ with $512 \times 512 \times 4$ cells. Periodic boundary conditions are applied in the direction perpendicular to $\vec{k}_{\text {saw }}$. The following magnetic parameters were used: $M_{s}=$ $50 \mathrm{kA} \mathrm{m}^{-1}$, Gilbert damping $\alpha=0.1, K_{2 \perp}=-8133 \mathrm{~J} \mathrm{~m}^{-3}$, $K_{2 \|}=800 \mathrm{~J} \cdot \mathrm{m}^{-3}$, exchange constant [49] $A_{\mathrm{ex}}=10^{-13} \mathrm{~J} \mathrm{~m}^{-1}$, cubic anisotropy $K_{c}=300 \mathrm{~J} \mathrm{~m}^{-3}$. MuMax on our operating system does not accomodate two uniaxial anisotropies, so only the in-plane uniaxial anisotropy was considered. Discarding the out-of-plane anisotropy $K_{2 \perp}$ effectively lowers the precession frequency and affects the in-plane and out-of-plane torques of the SAW on the magnetization. In this respect, quantitative comparisons between these simulations and the experiments should be made with great care. Acoustic parameters were taken as: $f_{\text {saw }}=990 \mathrm{MHz}$ and $V_{r}=2852 \mathrm{~m} \mathrm{~s}^{-1}$, which yields $\lambda_{\text {saw }}=2.8 \mu \mathrm{m}$. Anisotropy fluctuations were implemented by dividing the simulation window into $16 \times 16$ regions of coordinates $\vec{r}_{i}=\left(x_{i}, y_{i}\right)$, in which small random anisotropy variations proportionnal to $R$ could be added to the homogenous static anisotropies $K_{2 \|}$ and $K_{c}$. Using those same $16 \times 16$ regions, the effect of the propagating SAW was modelled by adding a time- and space varying component $\Delta K_{2 \|}\left(\vec{r}_{i}, t\right)$ (see 'Ku-dispersion. avi' movie in the supplementary information). Each region is $408 \mathrm{~nm} \approx \frac{\lambda_{\text {saw }}}{7}$ wide, a satisfyingly small fraction of the SAW wavelength. For $\vec{k}_{\text {saw }}$ along the easy axis, the final uniaxial anisotropy reads: $K_{2||}\left(\vec{r}_{i}, t\right)=K_{2||}$ $\left(1+\operatorname{randNorm}\left(\vec{r}_{i}\right) \mathrm{R}\right)+\Delta K_{2 \|}\left(1+\operatorname{randNorm}\left(\vec{r}_{i}\right) \mathrm{R}\right) \cos$ $\left(k_{\text {saw }} x_{i}-\omega t\right) H\left(t-t_{0}+\frac{x_{i}}{V_{r}}\right) H\left(-\left(t-t_{0}-\frac{x_{i}}{V_{r}}-\tau_{\text {saw }}\right)\right.$, with $\Delta K_{2 \|}=$ $200 \mathrm{~J} \mathrm{~m}^{-3}, \quad \tau_{\text {saw }}=10-12.5 \mathrm{~ns} \approx 10-12.5 T_{\text {saw }}$ the SAW pulse duration, $t_{0}=100 \mathrm{ps}$, and $R=0$ or 0.01 depending on whether anisotropy fluctuations are taken into account or not. The field is turned off at $t_{f}=17 \mathrm{~ns}$, a few ns after the SAW has completely left the simulation window. The simulation is then run for an extra 4 ns, i.e. over twice typical damping times in this material [49].

\section{ORCID iDs}

A Lemaître (으 https://orcid.org/0000-0003-1892-9726

L Thevenard (ㅇ https://orcid.org/0000-0002-4723-2955

\section{References}

[1] Back C H 1999 Science 285864

[2] Choi B C, Belov M, Hiebert W K, Ballentine G E and Freeman M R 2001 Phys. Rev. Lett. 86728
[3] Gerrits T, Van Den Berg H A M, Hohlfeld J, Bär L and Rasing T 2002 Nature 418509

[4] Hiebert W K, Lagae L and De Boeck J 2003 Phys. Rev. B 68020402

[5] Schumacher H W, Chappert C, Crozat P, Sousa R C, Freitas P P, Miltat J, Fassbender J and Hillebrands B 2003 Phys. Rev. Lett. 90017201

[6] Devolder T, Hayakawa J, Ito K, Takahashi H, Ikeda S, Crozat P, Zerounian N, Kim J-V, Chappert C and Ohno H 2008 Phys. Rev. Lett. 100057206

[7] Maunoury C, Devolder T, Lim C K, Crozat P, Chappert C, Wecker J and Bär L 2005 J. Appl. Phys. 97074503

[8] Krivorotov I N 2005 Science 307228

[9] Papusoi C, Delat B, Rodmacq B, Houssameddine D, Michel J P, Ebels U, Sousa R C, Buda-Prejbeanu L and Dieny B 2009 Appl. Phys. Lett. 95072506

[10] Devolder T, Tulapurkar A, Suzuki Y, Chappert C, Crozat P and Yagami K 2005 J. Appl. Phys. 98

[11] Vaysset A et al 2011 Appl. Phys. Lett. 98242511

[12] Zhang H, Hou Z, Zhang J, Zhang Z and Liu Y 2012 Appl. Phys. Lett. 100142409

[13] Bedau D, Liu H, Bouzaglou J-J, Kent A D, Sun J Z, Katine J A, Fullerton E E and Mangin S 2010 Appl. Phys. Lett. 96022514

[14] Shiota Y, Nozaki T, Bonell F, Murakami S, Shinjo T and Suzuki Y 2011 Nat. Mater. 1139

[15] Ghidini M, Pellicelli R, Prieto J L, Moya X, Soussi J, Briscoe J, Dunn S and Mathur N D 2013 Nat. Commun. 41453

[16] Stupakiewicz A, Szerenos K, Afanasiev D, Kirilyuk A and Kimel A V 2017 Nature $\mathbf{5 4 2}$ 71-4

[17] Thevenard L, Gourdon C, Prieur J-Y, von Bardeleben H J, Vincent S, Becerra L, Largeau L and Duquesne J-Y 2014 Phys. Rev. B 90094401

[18] Gowtham P G, Labanowski D and Salahuddin S 2016 Phys. Rev. B 94014436

[19] Dreher L, Weiler M, Pernpeintner M, Huebl H, Gross R, Brandt M and Goennenwein S 2012 Phys. Rev. B 86134415

[20] Kovalenko O, Pezeril T and Temnov V V 2013 Phys. Rev. Lett. 110266602

[21] Thevenard L, Duquesne J-Y, Peronne E, von Bardeleben H J, Jaffrès H, Ruttala S, George J-M, Lemaître A and Gourdon C 2013 Phys. Rev. B 87144402

[22] Thevenard L, Camara I S, Majrab S, Bernard M, Rovillain P, Lemaître A, Gourdon C and Duquesne J-Y 2016 Phys. Rev. B 93134430

[23] Li W, Buford B, Jander A and Dhagat P 2014 J. Appl. Phys. 115 17E307

[24] Balestriere P, Devolder T, Wunderlich J and Chappert C 2010 Appl. Phys. Lett. 96142504

[25] Elsen M, Boulle O, George J-M, Jaffrès H, Mattana R, Cros V, Fert A, Lemaître A, Giraud R and Faini G 2006 Phys. Rev. B 732

[26] Bihler C et al 2008 Phys. Rev. B 7845203

[27] Cormier M, Jeudy V, Niazi T, Lucot D, Granada M, Cibert J and Lemaître A 2014 Phys. Rev. B 90174418

[28] Linnik T, Scherbakov A, Yakovlev D, Liu X, Furdyna J and Bayer M 2011 Phys. Rev. B 84214432

[29] Schülein F J R, Zallo E, Atkinson P, Schmidt O G, Trotta R, Rastelli A, Wixforth A and Krenner H J 2015 Nat. Nanotechnol. 10512

[30] Labanowski D, Jung A and Salahuddin S 2016 Appl. Phys. Lett. 108022905

[31] Gowtham P G, Moriyama T, Ralph D C and Buhrman R A 2015 J. Appl. Phys. 118233910

[32] Thevenard L, Hussain S, von Bardeleben H J, Bernard M, Lemaître A and Gourdon C 2012 Phys. Rev. B 85064419 
[33] Thevenard L, Camara I S, Prieur J-Y, Rovillain P, Lemaître A, Gourdon C and Duquesne J-Y 2016 Phys. Rev. B 93140405

[34] Royer D and Dieulesaint E 2000 Elastic Waves in Solids I: Free and Guided Propagation (Advanced Texts in Physics) (Berlin: Springer)

[35] Gnatzig K, Dotsch H, Ye M and Brockmeyer A 1987 J. Appl. Phys. 624839

[36] Chen W, de Loubens G, Beaujour J-M L, Sun J Z and Kent A D 2009 Appl. Phys. Lett. 95172513

[37] Balestrière P, Devolder T, Kim J V, Lecoeur P, Wunderlich J, Novák V, Jungwirth T and Chappert C 2011 Appl. Phys. Lett. 99242505

[38] Mayergoyz I, Dimian M, Bertotti G and Serpico C 2004 J. Appl. Phys. 957004

[39] Devolder T and Chappert C 2004 Solid State Commun. 12997

[40] Devolder T and Chappert C 2004 J. Appl. Phys. 951933

[41] D'Aquino M, Perna S, Serpico C, Bertotti G, Mayergoyz I and Quercia A 2016 Physica B 486126
[42] Vansteenkiste A, Leliaert J, Dvornik M, Helsen M, GarciaSanchez F and Van Waeyenberge B 2014 AIP Adv. 4107133

[43] Thevenard L, Boutigny B, Güsken N, Becerra L, Ulysse C, Shihab S, Lemaître A, Kim J-V, Jeudy V and Gourdon C 2017 Phys. Rev. B 95054422

[44] Lee O J, Ralph D C and Buhrman R A 2011 Appl. Phys. Lett. 99102507

[45] Liu H, Bedau D, Backes D, Katine J A and Kent A D 2012 Appl. Phys. Lett. 101032403

[46] Park J, Ralph D C and Buhrman R A 2013 Appl. Phys. Lett. 103252406

[47] Largeau L, Camara I, Duquesne J-Y, Gourdon C, Rovillain P, Thevenard L and Croset B 2016 J. Appl. Crystallogr. 492073

[48] Camara I S, Croset B, Largeau L, Rovillain P, Thevenard L and Duquesne J-Y 2017 J. Appl. Phys. 121044503

[49] Shihab S, Riahi H, Thevenard L, von Bardeleben H J, Lemaître A and Gourdon C 2015 Appl. Phys. Lett. 106142408 